\title{
Comparing the effects of isoflurane and pentobarbital on the responses of cutaneous mechanoreceptive afferents
}

\author{
Ju-Wen Cheng ${ }^{1}$, Alison I Weber ${ }^{2}$ and Sliman J Bensmaia ${ }^{2 *}$
}

\begin{abstract}
Background: While pentobarbital has been used extensively in neurophysiological experiments investigating activity in peripheral nerves, it has fallen out of favor as an anesthetic because of safety concerns and is often replaced with isoflurane. However, the effects of isoflurane on the excitability of mechanoreceptive afferents have yet to be conclusively elucidated.

Methods: To fill this gap, we collected extracellular single-unit recordings of cutaneous mechanoreceptive afferents from the sciatic nerve of 21 rats during vibratory stimulation of the hindpaw. We then compared the strength and temporal structure of the afferent response measured under pentobarbital and isoflurane anesthesia.

Results: We found that the strength and temporal structure of afferent responses were statistically equivalent whether these were evoked under isoflurane or pentobarbital.

Conclusions: We conclude that, if these two anesthetics have any effect on the responses of mechanoreceptive afferents, their effects are indistinguishable.
\end{abstract}

Keywords: Mechanoreceptive afferents, Anesthesia, Isoflurane, Pentobarbital, Vibration, Entrainment

\section{Background}

Pentobarbital was the most commonly used anesthetic agent in neuroscience experiments in 2000-2001, but its use decreased from $33 \%$ in $2000-2001$ to $16 \%$ in $2005-$ 2006, while that of isoflurane and ketamine/xylazine increased ( 2 to $16 \%$ and 15 to $31 \%$, for isoflurane and ketamine/xylazine respectively) [1]. Previous studies have shown that these anesthetics cause disparate changes in cortical responses, but their effects on the sensory periphery, and on mechanoreceptive afferents in particular, remain to be conclusively elucidated. To fill this gap, we wished to compare the effects of pentobarbital and isoflurane on the responses of cutaneous mechanoreceptive afferents.

In experiments on Rhesus macaques (Macaca mulatta), a small dose of pentobarbital results in a considerable depression of both evoked potentials [2] and stimulus-

\footnotetext{
* Correspondence: sliman@uchicago.edu

${ }^{2}$ Department of Organismal Biology and Anatomy, University of Chicago,

Chicago, IL, USA

Full list of author information is available at the end of the article
}

evoked firing rates of cortical neurons [3]. Similarly, the addition of isoflurane to pre-existing anesthesia (nitrous oxide/narcotics) causes marked attenuation of motor evoked potential amplitudes [4], and somatosensory evoked potentials in response to electrical stimulation of the median nerve decrease in a dose-dependent manner with increasing concentrations of isoflurane [5,6]. When directly compared, the evidence suggests that isoflurane has a more profound impact on cortical responses than does pentobarbital [7].

Although the evidence that these two anesthetics affect cortical responses is unequivocal, their documented effects on the peripheral nervous system are not. Indeed, anesthetics appear to produce combinations of excitatory and depressant effects that are dependent on the preparation and/or type of neurons investigated. For example, pentobarbital reduces the amplitude of the early and late components of compound action potentials, associated with $\mathrm{A}$ and $\mathrm{C}$ fibers, respectively [8]. However, responses were evoked through electrical stimulation of the nerve, so could not be attributed to mechanoreceptive afferents. 
Isoflurane activates $C$ fiber nociceptors, depresses $A-\delta$ mechanoreceptive fibers [9] and results in a decrease in receptive field size and/or suppression of responses of spinal cord neurons [10] without affecting conduction velocities $[10,11]$. Isoflurane also reduces neuronal excitability in a small subset of trigeminal ganglion neurons of decerebrate guinea pigs [12] and of wide-dynamic-range and nociceptive specific neurons in the lumbar dorsal horn of goats [13].

In the present study, then, we sought to further characterize the effects of anesthesia on the responses of mechanoreceptive afferents. We used sinusoidal vibrations to compare the effects of two anesthetic regimes isoflurane and pentobarbital - on afferent excitability because the spiking behavior of afferents elicited by these stimuli has been extensively characterized [14]. Indeed, as afferents produce highly periodic responses to sinusoidal vibrations, using these stimuli allowed us to investigate the effects of anesthesia not just on the strength but also on the temporal patterning in afferent responses. First, we wished to determine whether changes in cortical responses caused by anesthesia might be due to its effects on afferent responses. Second, we wished to determine whether isoflurane was a suitable alternative to pentobarbital in neurophysiological experiments.

\section{Methods}

\section{Neurophysiological procedure}

All experimental procedures complied with the NIH Guide for the Care and Use of Laboratory Animals and were approved by the Animal Care and Use Committee of the University of Chicago. Experiments were conducted on 21 anesthetized Sprague-Dawley rats (Rattus norvegicus), weighing between 330 and 632 g. Anesthesia was induced with $4 \%$ inhalant isoflurane in an induction chamber; the animal was removed after loss of its righting reflex. To maintain anesthesia, 1.5$2 \%$ isoflurane in $100 \%$ oxygen was delivered via nose cone mask. Adapting a technique developed for primates [15], an incision roughly $4 \mathrm{~cm}$ long was made just posterior to the right femur, running parallel to the femur. The edges of the incision were then sutured to a plastic ring that was firmly secured to the table in order to create a skin-flap pool. The sciatic nerve was exposed by separating the biceps femoris muscle from the gluteus superficialis muscle in its aponeurosis. After isolating the sciatic nerve from the adjacent muscles, an 18mm-diameter laryngeal mirror was positioned under the nerve, and the basin formed by the skin flap was filled with saline to prevent the nerve from drying. The epineurium and perineurium of the nerve were peeled back sequentially with microforceps. A small nerve bundle was cut from the proximal portion of the nerve and placed on the mirrored platform. The saline in the skin-flap pool was then replaced with mineral oil to avoid signal shunting, and the nerve bundle $(\sim 250 \mu \mathrm{m}$ in diameter $)$ was carefully teased into smaller fiber bundles. Each of these smaller bundles was wrapped individually over a hooked silver wire recording electrode. Bundles were subdivided until action potentials from a single fiber were clearly isolated. Single unit potentials were amplified with an AC/DC differential amplifier (Model 3000, A-M Systems Inc., Sequim, WA), filtered using an equalizer (Ultragraph PRO FBQ3102, Behringer, Willich, Germany) and displayed using custom-made spike sorting software. Responses were elicited by gently brushing the rat paw with a cotton-tipped applicator. Once a single unit was isolated, the stimulating probe (diameter $=1 \mathrm{~mm}$ ) was fixed using cyanoacrylate to the point of maximum sensitivity (or hot spot) of its receptive field on the paw.

The stimulating probe was driven by a vibration exciter (Type ET-132-2, Labworks Inc., Costa Mesa, CA) driven by a linear power amplifier (Type PA-138, Labworks Inc., Costa Mesa, CA). The input voltage to the amplifier, under computer control, was delivered digitally by a multifunction data acquisition card (PCI-6229, National Instruments, Austin, TX; maximum single-channel output rate $=833 \mathrm{kHz}$; output rate used $=100 \mathrm{kHz}$ ). Probe movements were monitored using an accelerometer (Type 8702B50M1, Kistler Instrument Corporation, Amherst, NY) with a dynamic range of $\pm 50 \mathrm{~g}$. We used the side connector version of the accelerometer to facilitate mounting the accelerometer between the electrodynamic transducer and the stylus that delivered the stimulus to the skin. The threaded stud on the top of the accelerometer was mounted directly to the armature of the vibration exciter, and the stud of the stylus was attached to the bottom of the accelerometer. The output of the accelerometer was amplified and conditioned using a piezotron coupler (Type 5134A, Kistler Instrument Corporation, Amherst, NY), then read into the computer using a multifunction data acquisition card (PCI-6229, National Instruments, Austin, TX; maximum input rate $=250 \mathrm{kHz}$; input rate used $=100 \mathrm{kHz}$ ).

Units were classified as rapidly adapting (RA) or slowly adapting (SA) based on whether they produced transient or sustained responses to a sustained indentation. RA fibers were further subclassified as type I, having small and well-defined receptive fields, or type II, having large and poorly defined receptive fields. RA-II afferents, also known as Pacinian (PC) fibers, are also exquisitely sensitive to vibrations at higher frequencies and produce a vigorous response to air puffs.

\section{Experimental design}

Each experimental block, lasting 5 minutes, consisted of 10 repeated presentations of sinusoidal stimuli, each 
lasting 2 seconds, that varied in frequency and amplitude. The interstimulus interval was 0.1 second. Three different frequencies $(25,50$, and $100 \mathrm{~Hz})$ were tested, with 5 intensity levels at each frequency, spaced logarithmically $(100,177.8,316.2,562.3$, and $1000 \mu \mathrm{m}$, peak-to-peak, for $25 \mathrm{~Hz}$; 50, 88.9, 158.1, 281.2, and $500 \mu \mathrm{m}$ for $50 \mathrm{~Hz} ; 25,44.5,79.1,140.6$, and $250 \mu \mathrm{m}$ for $100 \mathrm{~Hz}$ ). The sinusoidal stimuli were ramped up and down with ramps lasting $50 \mathrm{~ms}$.

We recorded the responses of a single unit across two experimental blocks, separated by 30 minutes. In the control condition, both experimental blocks were recorded while the rat was under isoflurane anesthesia. This condition was included to assess the non-specific effects of prolonged stimulation on afferent responses. We could then quantify the degree to which responses during the second block were different from those in the first block and compare this difference to its counterpart in the experimental condition.

In the experimental condition, the first experimental block was identical to that in the control condition (i.e., the rat was under isoflurane anesthesia). After the first block, pentobarbital was injected intraperitoneally $(45 \mathrm{mg} / \mathrm{kg})$ and, 20 minutes later, isoflurane was discontinued. Oxygen administration was continued throughout the second block. After the end of the second experimental block, the isoflurane was restarted to prevent the animal from waking (pentobarbital anesthesia lasts approximately 20-60 minutes in rats, see ref. [16]), and the animal was euthanized by intraperitoneal injection of pentobarbital sodium and phenytoin sodium (Euthasol, Virbac Corporation, Fort Worth, TX) followed by exsanguination.

We adopted this experimental design (isoflurane anesthesia followed by either isoflurane or pentobarbital anesthesia) because recovery from pentobarbital anesthesia is very slow and somewhat unpredictable. It was therefore impractical to counterbalance the order in which anesthesia was delivered. The statistical analysis, described below, allowed us to disentangle the effects of the two anesthetics on the strength and patterning in neuronal responses.

\section{Statistical analyses}

Analyses were carried out using MATLAB (Mathworks Inc., Natick, MA). The effects of the different anesthesia regimes on afferent firing rates were analyzed using a three-way analysis of variance (ANOVA) with group (control or experimental), block number (1 or 2 ), and vibratory stimulus (one of three frequencies combined with one of five intensity levels) as factors. The critical factor, however, was the interaction between group and block number: Indeed, this interaction determines whether the difference (if any) between the responses in the first and second block is greater in the experimental or in the control group, and thus gauges the statistical reliability of the effect of anesthesia on afferent responses.

We also wished to assess whether anesthesia affected the temporal structure of afferent responses. Indeed, mechanoreceptive afferents exhibit highly stereotyped responses to sinusoidal stimuli; specifically, they produce one or a burst of action potentials within a restricted phase of each stimulus cycle, a patterning dubbed "entrainment." We sought to compare the effects of the two anesthetic regimes on afferent entrainment to sinusoidal stimulation using vector strength [17] as a measure of entrainment. Each spike can be considered as defining a vector of unit length with a phase angle $\theta i(0 \leqq \theta i<2 \pi)$ by taking $\theta i=2 \pi f t_{i}(\bmod 2 \pi)$, where $t_{i}$ is the timing of the spike, and $f$ is the stimulation frequency. Vector strength $r$ is given by:

$$
r=\frac{1}{n} \sqrt{\left(\sum_{i=1}^{n} \cos \theta i\right)^{2}+\left(\sum_{i=1}^{n} \sin \theta i\right)^{2}}
$$

Vector strength $r$ takes on the value of 1 if all of the spikes occur precisely at the same phase in each stimulus cycle and a value of 0 if spikes occur uniformly over the stimulus cycle. We tested whether anesthesia type had a significant effect on the vector strength using an ANOVA with group, block number, and vibratory stimulus as factors. Again, the critical factor was the interaction between group and block number.

\section{Results}

We recorded the responses of 21 single units (11 control group, 10 experimental group; 18 type I RA fibers, 3 SA fibers, see Figure 1 for sample spike traces). Given the

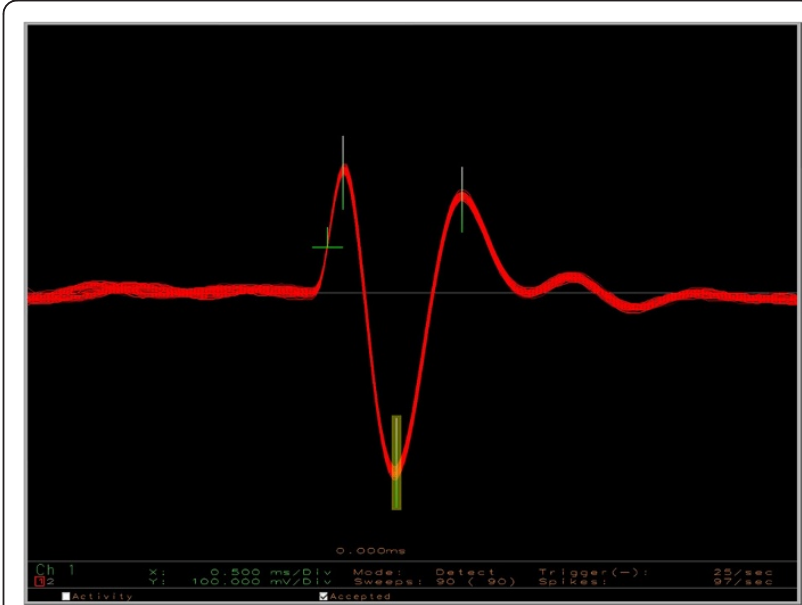

Figure 1 Neurophysiological recordings. An illustration of 90 (2-ms-long) action potential traces from a single unit displayed using custom-made spike sorting software (range of full screen: $\pm 1 \mathrm{~V}$ ). The signal to noise ratio is very high for these recordings. 
small number of SA fibers, we pooled data across fiber types, verifying that the two fiber types did not exhibit qualitatively different reactions to anesthesia. Figure 2 shows the mean firing rate of a single unit under anesthesia with isoflurane in the first and second experimental block (control condition). For this neuron, the responses were nearly identical in the two blocks. Figure 3 shows the mean firing rate of a (different) single unit under anesthesia with the first experimental block carried out under isoflurane anesthesia and the second under pentobarbital anesthesia (experimental condition). Again, the responses in the two blocks were not significantly different.

Because responses to vibratory stimuli vary widely across afferents, we normalized the firing rates of each afferent to then compare the effects of anesthesia across the entire population. Specifically, individual responses were transformed by subtracting from each response (i.e., the firing rate on each trial) the mean and dividing by the standard deviation of the responses across all stimulus conditions and experimental blocks (so that differences in firing rate across blocks would be preserved). This normalization removes any differences in the overall mean and standard deviation of the firing rates across afferents, leaving only differences across experimental blocks.

Figure 4 shows the normalized firing rates, averaged across all afferents, in the first block and second block, in the control and experimental conditions, respectively. Again, there was no systematic difference in firing rate between blocks for either group.

We also investigated the effects of anesthesia on the temporal structure of the response. Figure 5 shows the responses of a single afferent to a $50-\mathrm{Hz}$ sinusoid under
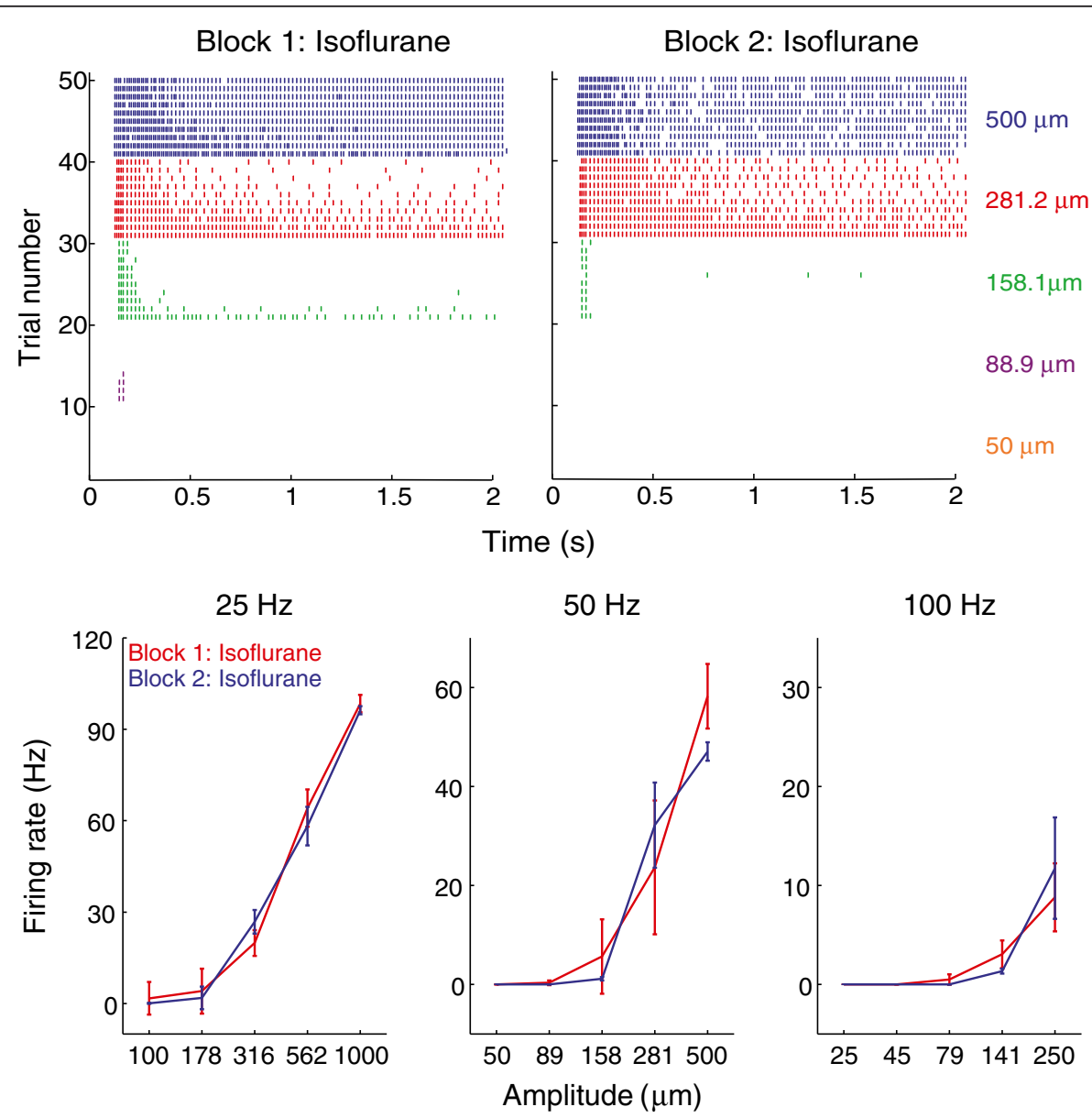

Figure 2 Typical responses of a single unit under anesthesia with isoflurane only. Top: Raster plots of the responses to 50-Hz stimulation in first (left) and second (right) blocks. Each row on the raster plots represents the spike train of the single unit corresponding to a given stimulus lasting for 2 seconds. Stimuli were presented ten times at each of five amplitudes (each indicated by a different color; at this frequency, the faintest stimulus did not elicit a response in this afferent). Bottom: Mean firing rates as a function of amplitude at the three frequencies tested. Each point indicates the average firing rate over 10 repetitions of each stimulus. Error bars denote the standard deviation. Note that we observed entrainment plateaus in a subset of fibers [14], but these were relatively uncommon given how sparsely amplitudes were sampled at each frequency. 


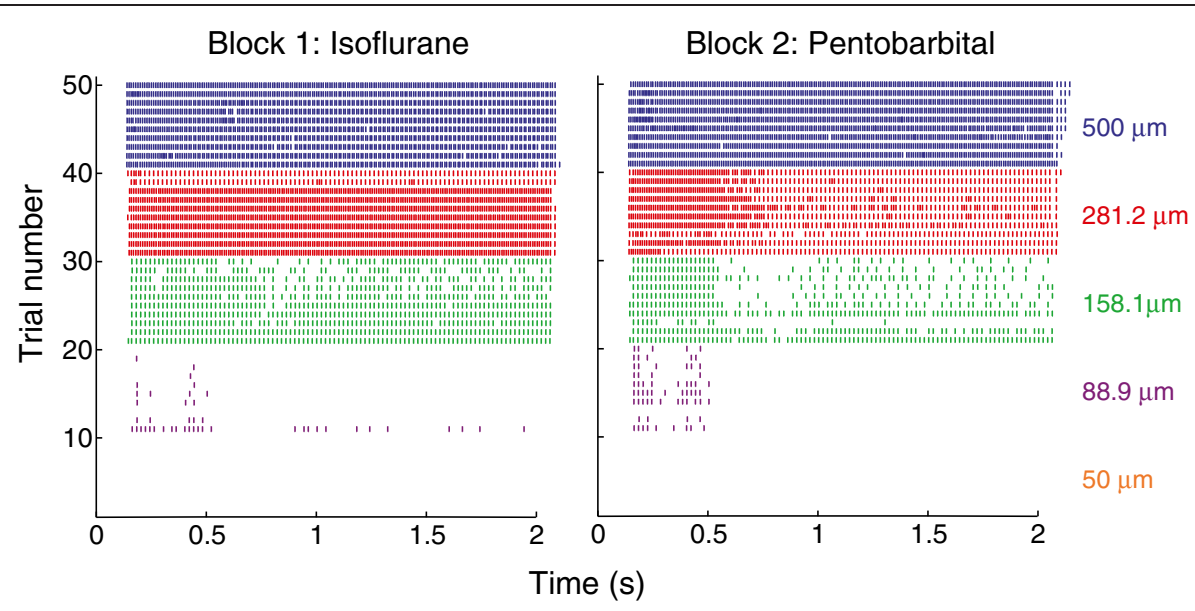

\section{$25 \mathrm{~Hz}$}

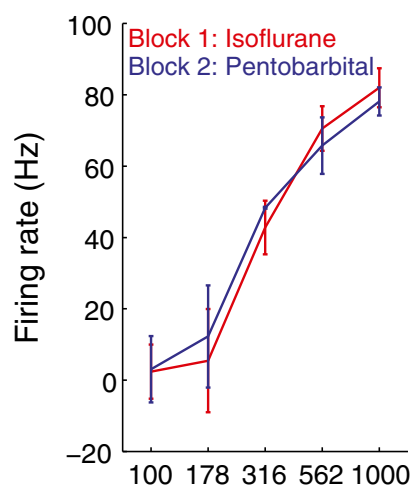

$50 \mathrm{~Hz}$

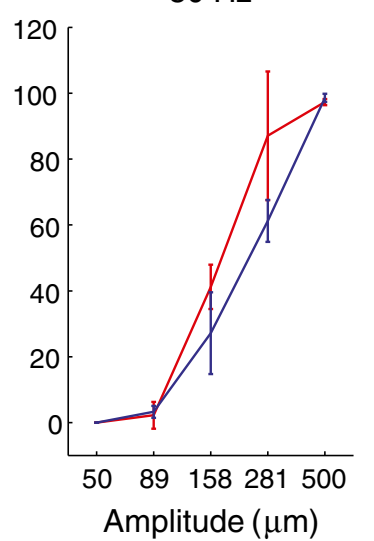

$100 \mathrm{~Hz}$

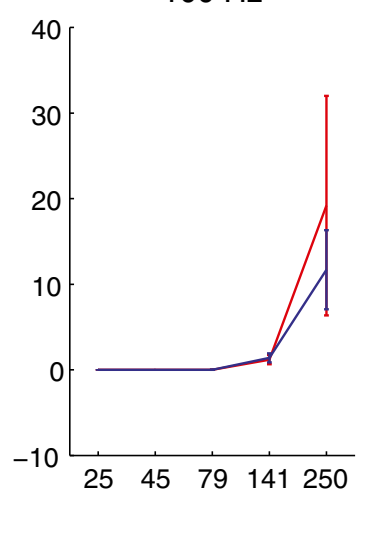

Figure 3 Typical responses of a single unit under anesthesia with first isoflurane and then pentobarbital. Top: Raster plots of the responses to $50-\mathrm{Hz}$ stimulation under isoflurane (left) and pentobarbital (right). Each row on the raster plots represents the spike train of the single unit corresponding to a given stimulus lasting for 2 seconds. Stimuli were presented ten times at each of five amplitudes. Bottom: Mean firing rates as a function of amplitude at the three frequencies tested. Error bars denote the standard deviation.

isoflurane anesthesia (red) and pentobarbital anesthesia (blue). As can be seen, the fine structure of the response is virtually identical under the two anesthetic regimes. Figure 6 shows the strength of entrainment of afferents in the first block and second block in the control and experimental conditions, respectively. There was no systematic effect of anesthesia on the temporal structure of the response across the afferent population (see ANOVA results below).

As expected, there was a significant effect of vibratory stimulus on both firing rate $(F(14,584)=103.2, \mathrm{p}<0.001)$ and vector strength $(F(14,584)=25.7, p<0.001)$. There was no significant difference in firing rate $(F(1,584)=1.33$, $p>0.2)$ or entrainment $(F(1,584)=0, p>0.5)$ across blocks. Importantly, however, the interaction between block number and group (control or experimental) was non-significant for both firing rate $(F(1,584)=0.5, p>0.4)$ and vector strength $(\mathrm{F}(1,584)=0.5, \mathrm{p}>0.05)$. In other words, the difference in response between the first and second blocks was not different when pentobarbital was used in the second block than when isoflurane was used. That is, anesthesia type did not have any effect on either the strength of or temporal patterning in the response. In conclusion, while the two anesthetic regimes may have affected the neuronal response, their effects were statistically indistinguishable.

\section{Discussion}

The main result in the present study is that isoflurane and pentobarbital do not differentially affect the responses of mechanoreceptive afferents. Indeed, both the strength of the afferent response and its fine temporal structure were statistically indistinguishable in the two conditions. Thus, differences in the effects of the two anesthetics on cortical responses cannot be attributed to differences in input from the periphery. Furthermore, the majority of neurophysiological experiments investigating the responses of mechanoreceptive afferents have been carried out using 


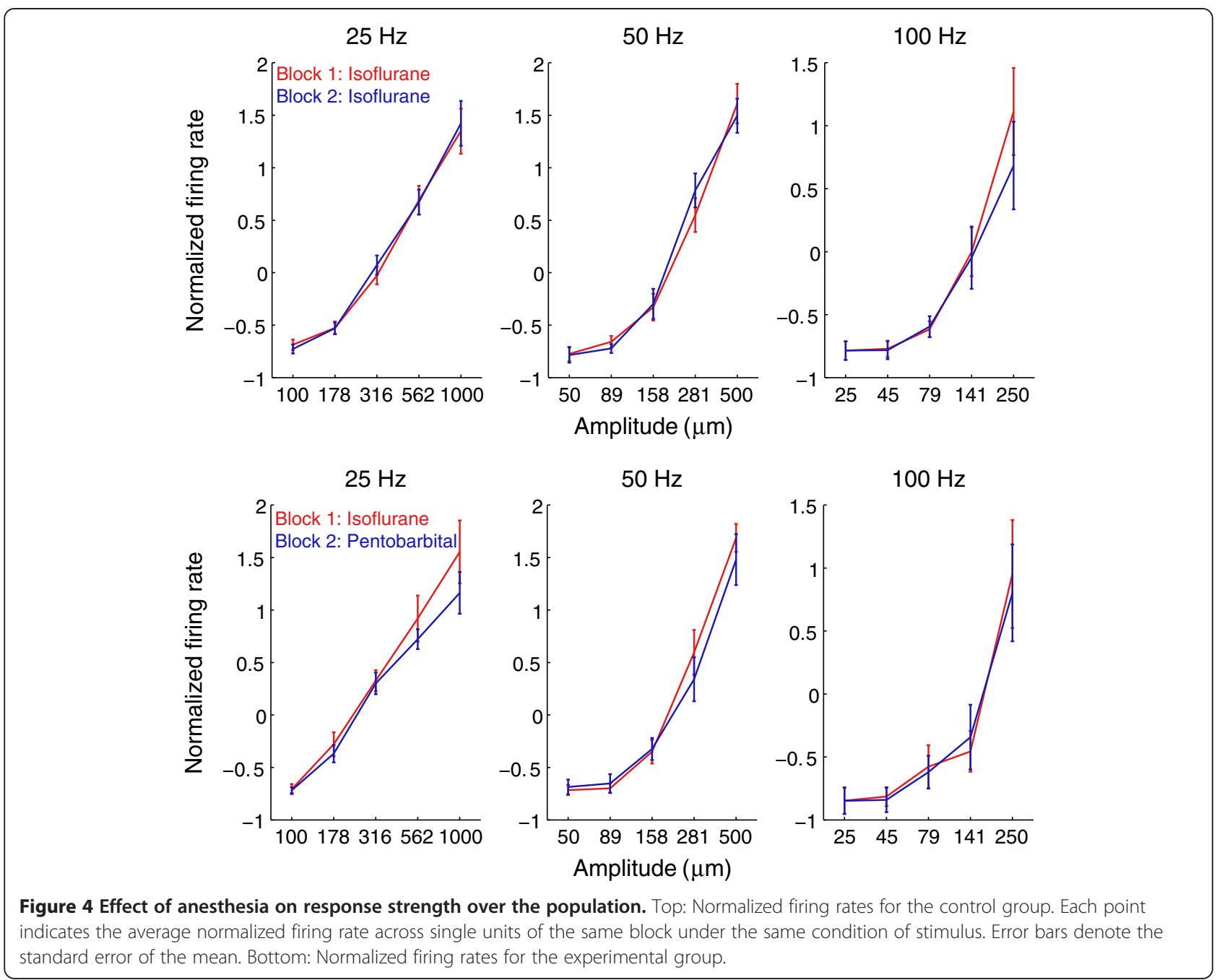

pentobarbital. The results of the present study suggest that isoflurane, a much safer anesthetic [18], yields identical results as does pentobarbital and so should be preferentially used in such experiments. Note that, although these experiments were carried out on cutaneous afferents of rats, mechanoreception in rodents, felines, and primates is highly homologous [19], so our results are likely to generalize across these mammalian orders.

\section{Residual isoflurane}

We wished to compare the effects of the two anesthetics on the responses of individual afferents, so it was necessary to maintain the isolation of the afferent signal through both experimental blocks and the inter-block interval. There was therefore a trade-off between extending the duration of the inter-block interval (to allow isoflurane to be eliminated) and maintaining the

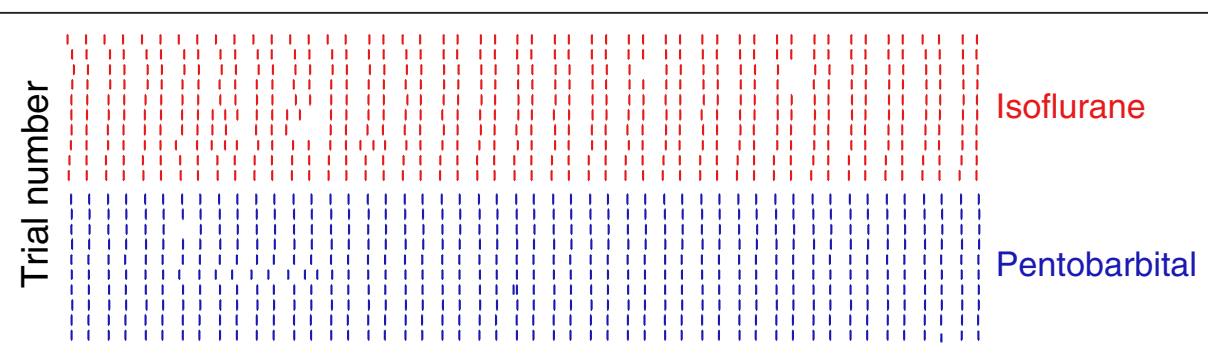

Figure 5 Entrainment of a single unit recorded in the experimental condition. Raster plots of the response evoked by $50-\mathrm{Hz}, 500-\mu \mathrm{m}$ sinusoidal vibration delivered for $0.5 \mathrm{~s}$. 

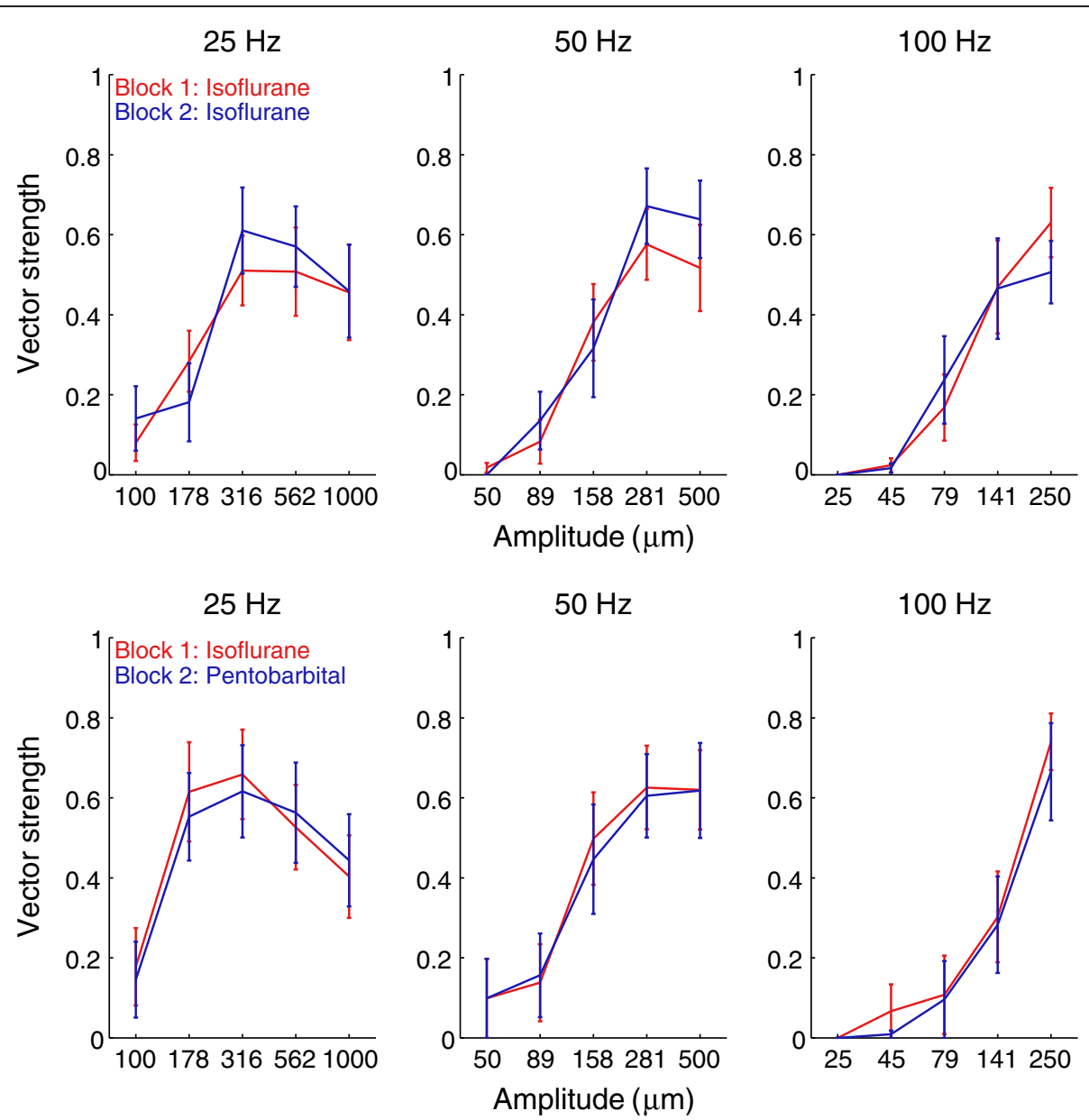

Figure 6 Effect of anesthesia on the temporal structure of the response over the population. Top: Entrainment in the control condition Each point indicates the mean vector strength across single units of the same block under the same condition of stimulus. Error bars denote the standard error of the mean. While the degree of entrainment is highly variable across afferents (as indicated by the large error bars), it is consistent across blocks. Bottom: Entrainment in the experimental group.

quality of recording from the neuron. We chose that duration to be 30 minutes. According to published data [20], approximately $52-72 \%$ of arterial isoflurane has been eliminated at this point in time in rats after an average of 4 hours of exposure to $1.8 \%$ isoflurane. In the current study, animals had been under anesthesia for an average of 2.5 hours by the end of the first experimental block, so the above estimate of residual isoflurane is likely an overestimate. In the experimental condition, responses in the second block thus reflected both the effects of pentobarbital and of some residual isoflurane. One possibility is that isoflurane has an effect on afferent responses that is relatively dose-independent. According to this view, measurements in the first and second block reflect an equivalent effect of isoflurane, and pentobarbital does not exert any additional effect on afferent responses.

\section{Molecular mechanisms}

At the periphery, isoflurane has been shown to inhibit neuronal $\mathrm{Ca}^{2+}$ channels through enhancement of current inactivation in rat dorsal root ganglion (DRG) neurons [21] but the behavioral relevance of these peripheral effects is unclear [22]. Pentobarbital may also lead to a reduction in the excitability of DRG neurons by suppressing the purinergic P2X receptors [23] and by activating $\mathrm{Cl}^{-}$ channels [24]. No study to date has investigated the extent to which these molecular mechanisms are pertinent to mechanoreceptive afferent neurons as the DRG comprises a wide variety of neurons, ranging from mechanoreceptive to thermoreceptive and nociceptive. Our results suggest that, to the extent that pentobarbital and isoflurane have an effect on mechanoreceptive afferent responses, their effects seem to be identical. Given the similarity in the responses measured in (non-anesthetized) humans using 
microneurography and those measured in macaques anesthetized with pentobarbital $[25,26]$, it is likely that isoflurane and pentobarbital have negligible effects on the responses of mechanoreceptive afferents.

\section{Conclusions}

We conclude that isoflurane and pentobarbital have identical (and probably negligible) effects on the responses of mechanoreceptive afferents. Indeed, both the strength and temporal structure of afferent responses to vibratory stimuli are indistinguishable under the two anesthetic regimes.

\section{Competing interests}

The authors declare that they have no competing interests.

\section{Authors' contributions}

JWC, AIW and SJB designed the experiments, JWC collected the data, JWC and AIW analyzed the data, JWC, AIW and SJB drafted the manuscript. All authors read and approved the final manuscript.

\section{Acknowledgments}

We would like to thank James Craig for comments on a previous version of this manuscript. This work was supported by NSF grant 1150209.

\section{Author details}

'Department of Physical Medicine and Rehabilitation, Chang Gung Memorial Hospital at Linkou, Taoyuan, Taiwan; Department of Organismal Biology and Anatomy, University of Chicago, Chicago, IL, USA. ${ }^{2}$ Department of Organismal Biology and Anatomy, University of Chicago, Chicago, IL, USA.

Received: 25 July 2012 Accepted: 16 April 2013

Published: 10 May 2013

\section{References}

1. Stokes EL, Flecknell PA, Richardson CA: Reported analgesic and anaesthetic administration to rodents undergoing experimental surgical procedures. Lab Anim 2009, 43(2):149-154.

2. Feldman S, Wagman IH: The Effect of Pentobarbital on Evoked Potentials in Brain of Macaca Mulatta. Electroencephalogr Clin Neurophysiol 1963, 15:747-760

3. Collins JG, Roppolo JR: Effects of pentobarbital and ethanol upon singleneuron activity in the primary somatosensory cortex of the rhesus monkey. J Pharmacol Exp Ther 1980, 213(2):337-345.

4. Calancie B, Klose K, Baier S, Green BA: Isoflurane-induced attenuation of motor evoked potentials caused by electrical motor cortex stimulation during surgery. J Neurosurg 1991, 74(6):897-904.

5. Mason DG, Higgins D, Boyd SG, Lloyd-Thomas AR: Effects of isoflurane anaesthesia on the median nerve somatosensory evoked potential in children. Br J Anaesth 1992, 69(6):562-566.

6. Samra SK, Vanderzant CW, Domer PA, Sackellares JC: Differential effects of isoflurane on human median nerve somatosensory evoked potentials. Anesthesiology 1987, 66(1):29-35.

7. Cheung SW, Nagarajan SS, Bedenbaugh PH, Schreiner CE, Wang X, Wong A: Auditory cortical neuron response differences under isoflurane versus pentobarbital anesthesia. Hear Res 2001, 156(1-2):115-127.

8. Tomsic M, Bajrovic F: Sensitivity of peripheral nerve fibres to sodium pentobarbital anaesthesia in rat. Pflugers Archiv : European journal of physiology 2000, 440(5 Suppl):R107-108.

9. Maclver MB, Tanelian DL: Volatile anesthetics excite mammalian nociceptor afferents recorded in vitro. Anesthesiology 1990, 72(6):1022-1030.

10. Vahle-Hinz C, Detsch O: What can in vivo electrophysiology in animal models tell us about mechanisms of anaesthesia? Br J Anaesth 2002, 89(1):123-142

11. Oh SS, Hayes JM, Sims-Robinson C, Sullivan KA, Feldman EL: The effects of anesthesia on measures of nerve conduction velocity in male C57BI6/J mice. Neurosci Lett 2010, 483(2):127-131.
12. Puil E, Gimbarzevsky B: Modifications in membrane properties of trigeminal sensory neurons during general anesthesia. J Neurophysiol 1987, 58(1):87-104.

13. Antognini JF, Carstens E, Tabo E, Buzin V: Effect of differential delivery of isoflurane to head and torso on lumbar dorsal horn activity. Anesthesiology 1998, 88(4):1055-1061.

14. Devecioglu I, Guclu B: Asymmetric response properties of rapidly adapting mechanoreceptive fibers in the rat glabrous skin. Somatosensory \& motor research 2012.

15. Talbot WH, Darian-Smith I, Kornhuber HH, Mountcastle VB: The sense of flutter-vibration: comparison of the human capacity with response patterns of mechanoreceptive afferents from the monkey hand. Journal of neurophysiology 1968, 31(2):301-334.

16. Fox JG, Anderson LC, Loew FM, Quimby FW (Eds): Laboratory Animal Medicine. London, UK: Academic Press; http://site.ebrary.com/id/10206370.

17. Grün S, Rotter S: Analysis of Parallel Spike Trains. Springer; 2010:59-67.

18. Sahaghian R, Faith NG, Czuprynski C: Comparison of systemic Listeria monocytogenes infection in esophageally inoculated mice anesthetized with isoflurane or pentobarbital. Lab animal 2009, 38(4):126-130.

19. Hunt SP, Koltzenburg M: The neurobiology of pain: (molecular and cellular neurobiology). Oxford University Press; 2005:2-3.

20. Chen M, Olsen JI, Stolk JA, Schweizer MP, Sha M, Ueda I: An in vivo $19 \mathrm{~F}$ NMR study of isoflurane elimination as a function of age in rat brain. NMR Biomed 1992, 5(3):121-126.

21. Kameyama K, Aono K, Kitamura K: Isoflurane inhibits neuronal Ca2+ channels through enhancement of current inactivation. $\mathrm{Br} J$ Anaesth 1999, 82(3):402-411.

22. Antognini JF, Kien ND: Potency (minimum alveolar anesthetic concentration) of isoflurane is independent of peripheral anesthetic effects. Anesth Analg 1995, 81(1):69-72.

23. Kitahara S, Yamashita M, lkemoto Y: Effects of pentobarbital on purinergic P2X receptors of rat dorsal root ganglion neurons. Can J Physiol Pharmacol 2003, 81(12):1085-1091.

24. Valeyev AY, Hackman JC, Holohean AM, Wood PM, Davidoff RA: Pentobarbital-activated $\mathrm{Cl}(-)$ channels in cultured adult and embryonic human DRG neurons. Brain Res Dev Brain Res 2000, 124(1-2):137-140.

25. Mountcastle VB: The sensory hand: neural mechanisms of somatic sensation. Harvard University Press; 2005.

26. Phillips JR, Johansson RS, Johnson KO: Responses of human mechanoreceptive afferents to embossed dot arrays scanned across fingerpad skin. The Journal of neuroscience : the official journal of the Society for Neuroscience 1992, 12(3):827-839.

doi:10.1186/1471-2253-13-10

Cite this article as: Cheng et al: Comparing the effects of isoflurane and pentobarbital on the responses of cutaneous mechanoreceptive afferents. BMC Anesthesiology 2013 13:10.

\section{Submit your next manuscript to BioMed Central and take full advantage of:}

- Convenient online submission

- Thorough peer review

- No space constraints or color figure charges

- Immediate publication on acceptance

- Inclusion in PubMed, CAS, Scopus and Google Scholar

- Research which is freely available for redistribution 